\title{
ELABORAÇÃO DE SORVETES FUNCIONAIS ADICIONADOS DE FRUTA EXÓTICA
}

\author{
MARINA ELIZABETH ALENCAR LIMA* \\ TAINÁ RIOS THIERS** \\ ARTHUR DE OLIVEIRA QUINTÃO*** \\ FERNANDA SENNA FERREIRA ${ }^{* * * * *}$ \\ LUCIANA RODRIGUES DA CUNHA ${ }^{\Phi}$ \\ SIMONE DE FÁTIMA VIANA DA CUNHA ${ }^{\Phi \Phi}$ \\ KELLY MOREIRA BEZERRA GANDRA $\Phi \Phi$ \\ SÍLVIA MENDONÇA VIEIRA $\Phi \Phi \Phi \Phi$ \\ REGINALDO DE SOUZA MONTEIRO" \\ PATRÍCIA APARECIDA PIMENTA PEREIRA ${ }^{\text {ax }}$
}

\begin{abstract}
Atualmente, os consumidores têm mostrado grande preocupação em adquirir produtos mais nutritivos e saudáveis, com sabores diferenciados e, acima de tudo, seguro para o consumo. Isso é uma tendência de mercado e, sendo o sorvete muito apreciado no Brasil, o objetivo deste estudo foi desenvolver e otimizar sorvetes funcionais adicionados de kinkan. Para este efeito, foram usadas três fibras dietéticas (amido modificado, polidextrose e inulina) isoladas e em diferentes combinações, a fim de se obter um produto rico em fibras e em compostos bioativos. Verificou-se que o kinkan tem boas propriedades físico-químicas, tanto em sua forma in natura e quanto processado, além de possuir elevados teores de antioxidantes. Estas características fazem com que o kinkan seja uma boa escolha para ser adicionado aos sorvetes rico em fibras. O sorvete funcional adicionado com kinkan demonstrou boa aceitabilidade, alta atividade antioxidante e alto teor de compostos fenólicos, o que o torna uma alternativa saudável e interessante para o mercado de alimentos funcionais. No que diz respeito as fibras utilizadas, podese concluir que, para uma melhor aceitabilidade do consumidor, deve ser adicionado inulina $(0,25 \%$ a $0,75 \%)$ e polidextrose $(0,25 \%$ a $0,75 \%$ ) na formulação dos sorvetes.
\end{abstract}

\section{PALAVRAS-CHAVE: KINKAN, SORVETE FUNCIONAL, FIBRA DIETÉTICA}

* Graduada em Ciência e Tecnologia de Alimentos, Departamento de Alimentos, Universidade Federal de Ouro Preto, Ouro Preto, MG, Brasil (e-mail: marina.eal@gmail.com).

** Graduanda em Ciência e Tecnologia de Alimentos, Departamento de Alimentos, Universidade Federal de Ouro Preto, Ouro Preto, MG, Brasil (e-mail: tainariost@gmail.com).

*** Graduando em Ciência e Tecnologia de Alimentos, Departamento de Alimentos, Universidade Federal de Ouro Preto, Ouro Preto, MG, Brasil (e-mail: arthurquintao2@hotmail.com).

**** Mestranda em Biotecnologia, Núcleo de Pesquisas em Ciências Biológicas, Universidade Federal de Ouro Preto, Ouro Preto, MG, Brasil (e-mail: fernandasenna88@hotmail.com).

Ф Doutora em Ciência e Tecnologia de Alimentos, Departamento de Alimentos, Universidade Federal de Ouro Preto, Ouro Preto, MG, Brasil (e-mail: Ircunhaufv@yahoo.com.br).

Ф Ф Doutora em Ciências Biológicas, Departamento de Alimentos, Universidade Federal de Ouro Preto, Ouro Preto, MG, Brasil (e-mail: simonenutricao@yahoo.com.br).

ФФФ Doutora em Tecnologia de Alimentos, Departamento de Alimentos, Universidade Federal de Ouro Preto, Ouro Preto, MG, Brasil (e-mail: kelly_gandra@yahoo.com.br).

$\Phi \Phi \Phi \Phi$ Doutora em Ciência dos Alimentos, Departamento de Alimentos, Universidade Federal de Ouro Preto, Ouro Preto, MG, Brasil (e-mail: silviamvieira@yahoo.com.br).

a Mestrando em Saúde e Nutrição, Escola de Nutrição, Departamento de Alimentos, Universidade Federal de Ouro Preto, Ouro Preto, MG, Brasil (e-mail: reginaldo_monteiro@yahoo.com.br).

a Doutora em Ciência dos Alimentos, Departamento de Alimentos, Universidade Federal de Ouro Preto, Ouro Preto, MG, Brasil (e-mail: patriciaaparecidapimentapereira@yahoo.com.br). 


\section{INTRODUÇÃO}

De acordo com a portaria $n^{\circ} 226$ de 2005 , gelados comestíveis são produtos alimentícios obtidos a partir de uma emulsão de gorduras e proteínas, com ou sem adição de outros ingredientes e substâncias, ou de uma mistura de água, açúcares e outros ingredientes e substâncias que tenham sido submetidas ao congelamento, em condições tais que garantam a conservação do produto no estado congelado ou parcialmente congelado, durante a armazenagem, o transporte e a entrega ao consumidor (BRASIL, 2005). Atualmente, a indústria sorveteira está em destaque e há grande perspectiva de crescimento da área, visto que o consumo de sorvete no Brasil cresceu $81,6 \%$ nos últimos 10 anos (ABIS, 2013).

Os ingredientes básicos de sobremesas congeladas podem ser agrupados em produtos lácteos e não lácteos. Os produtos lácteos formam ingredientes básicos que fornecem gordura e sólidos não gordurosos do leite (SNGL). Entre os produtos não lácteos encontram-se adoçantes, estabilizantes/emulsificantes, ovos, frutas, nozes, essências, produtos especiais e água (Marshall \& Arbucle, 1996).

O avanço dos conhecimentos sobre a relação entre alimentação e saúde, bem como os elevados custos da saúde pública e a busca permanente da indústria por inovações têm gerado novos produtos, cujas funções pretendem ir além do conhecido papel nutricional dos alimentos. Pesquisas têm sido realizadas visando à identificação de novos compostos bioativos e o estabelecimento de bases científicas para a comprovação das alegações de propriedades funcionais dos alimentos (Seolin et al., 2013).

O mercado para esse tipo de alimento movimenta cerca de US\$ 60 bilhões no mundo, responsável por mais da metade dos investimentos publicitários na área alimentícia e com expectativas de crescimento da ordem de $5 \%$ ao ano. Trata-se, portanto, de um segmento de grande interesse para as indústrias alimentícias, que têm buscado explorar a relação entre o consumo de determinados ingredientes com a redução de fatores de risco para doenças específicas, principalmente as crônicas não transmissíveis, ou a melhoria do desempenho físico ou mental (Sales et al.,2008).

Neste contexto, surge uma alternativa para a elaboração de produtos que atendam essas exigências do consumidor a adição de nutrientes funcionais benéficos à saúde tais como as fibras. As fibras insolúveis produzem redução no tempo de trânsito intestinal e aumento do volume fecal levando à diminuição da constipação intestinal. As fibras solúveis provocam retardo no esvaziamento gástrico, na absorção da glicose e do colesterol, permitindo melhor controle dos níveis plasmáticos, promovendo redução do risco de doenças cardiovasculares e diabetes, além de contribuir com a saciedade (Cummings et al., 2004).

A polidextrose é um polissacarídeo extremamente estável dentro de uma faixa ampla de $\mathrm{pH}$, temperatura e condições de processamento e estocagem. É sintetizada pela polimerização randômica da glicose e pode ser considerada um alimento funcional. É tolerada uma média de $90 \mathrm{~g}$ por dia, sem efeitos laxativos (Paucar-Menacho et al., 2008). Já o amido resistente é a fração que não fornecerá glicose ao organismo, mas que será fermentado no intestino grosso levando a formação de gases e ácidos graxos de cadeia curta. Devido a esta característica, considera-se que os efeitos do amido resistente sejam, em alguns casos, comparáveis aos das fibras alimentares e, por este motivo é considerado componente desta (Walter et al., 2005).

A inulina é um carboidrato que tem sido designado como prebióticos e fibras alimentares solúveis, por sua não digestibilidade pelas enzimas do trato digestivo humano, estimulo seletivo do crescimento e atividade de bactérias intestinais promotoras de saúde (Moscatto et al., 2004).

Além da adição de compostos com a finalidade de aumentar o valor nutritivo, o aproveitamento de espécies frutíferas exóticas reflete na oferta de novas alternativas de frutas frescas para consumo e matéria-prima para agroindústria, constituindo uma importante fonte de alimentos (Nascimento et al., 2008). Essas frutas são ricas em vitaminas, sais minerais e compostos 
bioativos. O interesse por frutas exóticas tem crescido rapidamente devido a busca por novos produtos e sabores incentivando. Portanto, os pequenos produtores (Rogez et al., 2004).

O kinkan ou kumquat (Fortunella margarita) se encaixa nesse perfil, sendo uma fruta cítrica originaria da China, da família Rutaceae. As frutas e folhas da Fortunella são usadas na medicina chinesa há tempos e recentemente estudos têm sido realizados para estudar os efeitos de seus compostos bioativos (Sadek et al.,2009; Shun-zhen et al., 2012; Peng et al., 2013).

Segundo Sadek et al. (2009), o kinkan possui alto teor de flavonóides, que favorecem a diminuição da pressão arterial. Outros estudos indicam também que ele auxilia no combate a obesidade e doenças relacionadas (Tanet al., 2014), além de prevenir o câncer de próstata (Jayaprakasha et al., 2012).

Diante disso, o objetivo deste trabalho foi elaborar e otimizar sorvetes funcionais adicionados de kinkan.

\section{MATERIAL E MÉTODOS}

\subsection{MATERIAL}

As matérias-primas utilizadas foram: leite UHT, creme de leite, sacarose, mix de estabilizantes/emulsificantes (Gemacon $\AA$ ), polidextrose (Nutramax $®$ ), inulina (Metachem $®$ ), amido resistente (Gemacon®) e kinkan.

O kinkan foi adquirido em supermercado local da cidade de Ouro Preto - MG, e processado sob condições adequadas de higiene no laboratório de Técnica Dietética da Escola de Nutrição na Universidade Federal de Ouro Preto.

Os frutos foram lavados em água corrente para remoção das sujidades e imersos em solução de hipoclorito de sódio a 2,5\% por 15 minutos. Após processo de higienização, os frutos foram selecionados de modo a descartar as frutas impróprias para utilização. Em seguida, estes foram homogeneizados em liquidificador industrial com $80 \%$ de água (concentração escolhida por meio de testes prévios), sendo armazenada em recipientes plásticos e estocada em ultrafreezer a $-50^{\circ} \mathrm{C}$.

\subsection{ELABORAÇÃO DOS SORVETES}

Para a elaboração dos sorvetes foi utilizado planejamento simplex-lattice (Cornell, 1983) para avaliar os efeitos e para otimizar as proporções de amido resistente $\left(X_{1}\right)$, polidextrose $\left(X_{2}\right)$ e inulina $\left(\mathrm{X}_{3}\right)$. O planejamento experimental e seus níveis encontram-se na Tabela 1.

Foram elaborados sorvetes tradicionais adicionados de polpa de kinkan utilizando três tipos de fibras alimentícias comerciais (amido modificado, polidextrose e inulina) isoladas e em diferentes combinações.

O presente estudo foi analisado e aprovado pelo Comitê de Ética em Pesquisa $\mathrm{n}^{\circ} 32931914.5 .0000 .5150$.

A gordura foi substituída por $10 \%$ de fibras, que foi definida por meio de testes prévios de acordo com as legislações vigentes.

Cada formulação foi elaborada contendo $5,76 \%$ de creme de leite, $41 \%$ de leite UHT, 9,52\% de sacarose e $0,39 \%$ de mix de estabilizantes e emulsificantes. Os ingredientes foram dispersos, sob agitação, com o auxílio de um liquidificador industrial. As fibras isoladas e em diferentes combinações foram misturadas à sacarose e ao mix de estabilizantes e emulsificantes para facilitar a solubilização. Adicionou-se $33,33 \%$ da polpa do kinkan a base do sorvete. Em seguida este foi misturado em liquidificador e a mistura foi levada a sorveteira semi-industrial, até obter consistência desejada. $\mathrm{O}$ sorvete foi armazenado em ultrafreezer a $-40^{\circ} \mathrm{C}$ para posteriores análises. 


\section{TABELA 1 - PLANEJAMENTO EXPERIMENTAL SIMPLEX-LATTICE DAS DIFERENTES FORMULAÇÕES DE SORVETES}

\begin{tabular}{|c|c|c|c|}
\hline \multirow{2}{*}{ Tratamentos } & \multicolumn{3}{|c|}{ Variáveis } \\
\cline { 2 - 4 } & $\mathbf{X}_{1}$ & $\mathbf{X}_{2}$ & $\mathbf{X}_{3}$ \\
\hline 1 & 1 & 0 & 0 \\
\hline 2 & 0 & 1 & 0 \\
\hline 3 & 0 & 0 & 0 \\
\hline 4 & 0,5 & 0,5 & 0,5 \\
\hline 5 & 0,5 & 0 & 0,5 \\
\hline 6 & 0 & 0,5 & 0,16 \\
\hline 7 & 0,68 & 0,16 & 0,16 \\
\hline 8 & 0,16 & 0,68 & 0,68 \\
\hline 9 & 0,16 & 0,16 & 0,34 \\
\hline 10 & 0,33 & 0,33 & \\
\hline
\end{tabular}

\subsection{ANÁLISES REALIZADAS}

Para caracterização do kinkan in natura foram realizadas análises de umidade, lipídeos, cinzas, proteínas e acidez total (AOAC, 2000), pH e sólidos solúveis (IAL, 2005), fenólicos totais pelo método Folin-Ciocalteu (Waterhouse, 2002) e atividade antioxidante (Rufino et al., 2007).

Além disso, foram realizadas análises de pH e sólidos solúveis (IAL, 2005), acidez total (AOAC, 2000), fenólicos totais pelo método Folin-Ciocalteu (Waterhouse, 2002) e atividade antioxidante (Rufino et al., 2007) na polpa processada com $80 \%$ de água.

Já para os sorvetes elaborados a partir desta matéria prima, foram realizadas análises de umidade, cinzas, e acidez total (AOAC, 2000), pH e sólidos solúveis (IAL, 2005), fenólicos totais pelo método Folin-Ciocalteu (Waterhouse, 2002) e atividade antioxidante (Rufino et al., 2007).

Para a realização das análises de compostos fenólicos e atividade antioxidante foram obtidos extratos para o kinkan in natura, para o kinkan processado e para as diferentes formulações de sorvete de acordo com metodologia proposta por Larrauri et al (1997). As amostras foram pesadas em tubos de centrífuga e extraídos seqüencialmente com $40 \mathrm{~mL}$ de metanol/água (50:50, v/v) por $1 \mathrm{~h}$. Os tubos foram então centrifugados a $25.400 \mathrm{~g}$ por $15 \mathrm{~min}$ e o sobrenadante será recuperado. Em seguida, $40 \mathrm{~mL}$ de acetona/água $(70: 30$, v/v) foi adicionado ao resíduo, e após extração por 60 min os tubos foram submetidos novamente a centrifugação a $25.400 \mathrm{~g}$ por $15 \mathrm{~min}$. No extrato obtido após a segunda centrifugação foi adicionado de água destilada, até perfazer o volume de $100 \mathrm{~mL}$.

A análise sensorial foi realizada no Laboratório de Análise Sensorial da Universidade Federal de Ouro Preto. $O$ teste de aceitação com consumidor foi realizado em relação aos atributos sabor, doçura, consistência e impressão global usando escala hedônica de 9 pontos ( 1 = desgostei extremamente, 9 = gostei extremamente) (Stone \&Sidel, 1985). O teste foi conduzido com 100 consumidores de sorvete. A avaliação sensorial foi realizada em duas sessões (cinco amostras por sessão). Aproximadamente $5 \mathrm{~g}$ de cada amostra foram servidas a cada consumidor (Acosta et al., 2008). Os sorvetes foram mantidos em freezer a $-18^{\circ} \mathrm{C}$ e servidos assim que retirados deste em copos plásticos brancos descartáveis com capacidade para $50 \mathrm{~mL}$ e codificados por três dígitos aleatórios.

\subsection{AVALIAÇÃO DOS RESULTADOS}

Inicialmente, foram realizadas as médias das avaliações físico-químicas do kinkan in natura e da polpa (80\% de água) em software Excel (2010). 
Além disso, realizou-se análise estatística baseada no modelo predito para as avaliações físico-químicas e sensoriais das diferentes formulações de sorvete. Para avaliar o ajuste dos dados, observou-se a análise de variância e o coeficiente de determinação $\left(R^{2}\right)$ de cada parâmetro analisado em software Statistica 6.0 (StatSoft Inc., U.S. A., 2001). Nos parâmetros onde não houve ajuste de modelo, fez-se teste de médias (Scott-Knott) a $5 \%$ de probabilidade em software Sisvar (Ferreira, 2000).

\section{RESULTADOS E DISCUSSÃO}

Os resultados das análises físico-químicas do kinkan in natura e processado encontram-se na Tabela 2.

TABELA 2 - AVALIAÇÃO FÍSICO-QUÍMICA DO KINKAN IN NATURA E PROCESSADO

\begin{tabular}{|c|c|}
\hline \multicolumn{2}{|l|}{ Kinkan in natura } \\
\hline $\mathrm{pH}$ & $4,2 \pm 0,0$ \\
\hline Acidez Total (g ácido cítrico/100 g) & $0,3 \pm 0,0$ \\
\hline Umidade ( $\mathrm{g} / 100 \mathrm{~g}$ de fruta) & $84,5 \pm 0,3$ \\
\hline Lipídios (g/100 g de fruta) & $0,1 \pm 0,1$ \\
\hline Cinzas (g/100 g de fruta) & $0,7 \pm 0,0$ \\
\hline Proteína ( $\mathrm{g} / 100 \mathrm{~g}$ de fruta) & $0,6 \pm 0,1$ \\
\hline Teor de sólidos solúveis ( $\left.{ }^{\circ} \mathrm{Brix}\right)$ & $12,4 \pm 0,3$ \\
\hline Fenólicos totais (mg GAE/ g de fruta) & $87,3 \pm 12,4$ \\
\hline Atividade antioxidante-DPPH $\left(\mathrm{EC}_{50}-\mathrm{g}\right.$ fruta/g DPPH) & $36905,04 \pm 14891,9$ \\
\hline \multicolumn{2}{|c|}{ Kinkan processado ( $80 \%$ de água) } \\
\hline $\mathrm{pH}$ & $4,8 \pm 0,0$ \\
\hline Acidez Total (g ácido cítrico/100 g) & $0,1 \pm 0,0$ \\
\hline Teor de sólidos solúveis ( ${ }^{\circ}$ Brix $)$ & $9,2 \pm 0,1$ \\
\hline Fenólicos totais (mg GAE/ g da fruta) & $58,6 \pm 8,1$ \\
\hline Atividade antioxidante-DPPH $\left(\mathrm{EC}_{50}-\mathrm{g}\right.$ fruta/g DPPH) & $33395,6 \pm 5270,6$ \\
\hline
\end{tabular}

Sendo o kinkan da família Rutaceae (Sadek et al., 2009), a mesma das frutas cítricas, como laranja, tangerina e limão (Flora do Brasil, 2008), é plausível compará-lo à essas frutas.

Pode-se observar pela Tabela 2 que tanto para o kinkan in natura quanto para o kinkan processado os valores de $\mathrm{pH}$ foram semelhantes $(4,2$ e 4,8 , respectivamente). Segundo Pereira (2009), os valores de pH para a tangerina e para a laranja são 4,2 e 4,1, respectivamente. Isso mostra que o kinkan apresenta $\mathrm{pH}$ similar a estas frutas. Em relação à acidez total, o kinkan in natura obteve valor de $0,3 \%$ (Tabela 2). Segundo estudos de Pereira (2009) sobre a qualidade de frutas tropicais cítricas produzidas no Ceará, a acidez titulável da laranja variou entre 0,4 e 0,5\%, e a tangerina obteve um teor de $0,4 \%$. Devido a acidez total do kinkan in natura ter sido $0,3 \%$, este pode ser caracterizado como uma fruta menos ácida do que as outras citrus. A acidez é um parâmetro de grande influência sensorial, especialmente para sabor e aroma, pois segundo Júnior et al. (2010) esta influência o escurecimento oxidativo dos tecidos vegetais, sendo que menor acidez faz com 
que a velocidade de escurecimento do fruto seja maior. Para o kinkan processado, o valor de acidez total foi de $0,1 \%$. Esta diminuição nos valores pode ser em decorrência a adição de água à polpa, na qual diluiu os componentes da fruta.

O teor de sólidos solúveis indica o grau de maturidade e determina a qualidade da fruta, exercendo importante papel no sabor (Vilas Boas et al., 2004), principalmente para o comércio de frutas in natura, uma vez que, segundo Resende et al. (2010) os consumidores preferem frutos mais doces. Segundo o Centro de Qualidade em Horticultura da CEAGESP, o teor mínimo de sólidos solúveis para laranjas deve ser entre 9,0 e $10^{\circ} \mathrm{Brix}$ e para tangerina, entre 9,0 e 10,5 ${ }^{\circ} \mathrm{Brix}$. No presente estudo, o kinkan in natura apresentou teor de sólidos solúveis acima destas faixas $(12,4$ ${ }^{\circ}$ Brix) (Tabela 2), podendo assim, agradar os consumidores. Já o kinkan processado apresentou teor menor $\left(9,2^{\circ}\right.$ Brix $)$, fato que pode ser explicado pela adição de água à polpa.

O resultado obtido para a umidade $(84,5 \%)$ mostra que o kinkan é uma fruta com teor de umidade semelhante ao da laranja $(85,4 \%)$, da tangerina $(89,2 \%)$ e do limão $(87,4 \%)$ (TACO, 2011). Porém, possui maior conteúdo de cinzas $(0,7 \%)$, uma vez que a laranja tem teor de $0,5 \%$, a tangerina $0,3 \%$ e o limão $0,4 \%$. Segundo Uchoa et al. (2008) altas taxas de cinzas podem estar associadas a uma maior concentração dos minerais. Isso mostra que o kinkan pode possuir maior concentração de minerais do que outras frutas cítricas como laranja e tangerina.

O teor de lipídios foi de $0,15 \%$ para o kinkan in natura (Tabela 2), próximo aos das outras frutas cítricas $(0,1$ a $0,2 \%)$ (TACO, 2011). O teor de proteína foi de $0,6 \%$, também está próximo aos de outras frutas cítricas, como laranja $(0,7 \%)$ e tangerina $(0,8 \%)$, distanciado-se apenas do limão $(0,9 \%)$ (TACO, 2011).

De acordo com os resultados físico-químicos, pode-se afirmar que as propriedades do kinkan coincidem com as propriedades das outras frutas cítricas, além de ter propriedades desejáveis sensorialmente, como elevados teores de sólidos solúveis e a baixa acidez.

O conteúdo de fenólicos totais variou de 58,6 (kinkan processado) a 87,3mg GAE/g de fruta (kinkan in natura) (Tabela 2). De acordo com Vasco et al. (2008), pode-se classificar os polifenóis em baixo (<1 mg GAE/ g), médio (1-5 mg GAE/ g) e alto ( $>5 \mathrm{mg} \mathrm{GAE} \mathrm{/} \mathrm{g)} \mathrm{teor.} \mathrm{Diante} \mathrm{disso,}$ observa-se que tanto o kinkan in natura quanto o processado podem ser classificados como tendo elevada concentração de fenóis. Esta classificação indica que a presente fruta é uma excelente fonte de fenóis e que, mesmo durante seu processamento, as perdas não fazem com que se tenham prejuízos em relação a este composto bioativo. Os compostos fenólicos do kinkan vêm sendo amplamente estudados (Agócs et al., 2007; Sadek et al., 2009; Barreca et al., 2011), devido ao fato de que, atualmente, os compostos bioativos vem recebendo maior atenção dos consumidores (Barreca et al., 2011). As análises de Sadek et al. (2011) para a variedade Grega de Kinkan obteve resultado de conteúdo fenólico de $77,4 \mathrm{mg} \mathrm{GAE} / \mathrm{g}$ da fruta e para a variedade egípcia, 106,2 mg $\mathrm{GAE} / \mathrm{g}$ de fruta. O teor de fenólicos no Kinkan analisado neste estudo(87,3 mg GAE/ g de fruta), correspondem aos encontrados por Sadek et al. (2009). Observa-se também que os valores da atividade antioxidante tanto do kinkan in natura quanto o processado foram semelhantes $(36905,4$ e 33395,6, respectivamente). Entretanto, optou-se por não comparar estes dados com os dados obtidos por outros estudos com kinkan para a atividade antioxidante, devido às diferenças entre os métodos e apresentação dos resultados, que não permitem uma comparação adequada.

Não houve ajuste do modelo para $\mathrm{pH}$, acidez total, lipídeos, umidade, cinzas e fenólicos totais. Diante disso foi feito teste de médias (Tabela 3).

A acidez total, o conteúdo de fenólicos totais e os teores de cinzas não apresentaram diferenças significativas entre as formulações $(p>0,05)$.

O teor de umidade (Tabela 3 ) encontrado nas diferentes formulações dos sorvetes variou de $67 \%$ a $71 \%$. 
TABELA 3 - AVALIAÇÃO PH, ACIDEZ TOTAL, LIPÍDEOS, UMIDADE, CINZAS E FENÓLICOS TOTAIS DOS SORVETES FUNCIONAIS ADICIONAIS DE KINKAN

\begin{tabular}{cccccc}
\hline Formulação & pH & Acidez Total & Umidade & Cinzas & Fenólicos Totais \\
\hline $\mathbf{1}$ & $5,95 \pm 0,0 \mathrm{f}$ & $0,02 \pm 0,0 \mathrm{a}$ & $71,3 \pm 2,9 \mathrm{a}$ & $0,5 \pm 0,0 \mathrm{a}$ & $58,6 \pm 11,1 \mathrm{a}$ \\
$\mathbf{2}$ & $6,03 \pm 0,0 \mathrm{a}$ & $0,02 \pm 0,0 \mathrm{a}$ & $68,7 \pm 0,7 \mathrm{~b}$ & $0,5 \pm 0,1 \mathrm{a}$ & $54,3 \pm 13,9 \mathrm{a}$ \\
$\mathbf{3}$ & $6,02 \pm 0,0 \mathrm{~b}$ & $0,02 \pm 0,0 \mathrm{a}$ & $67,4 \pm 1,2 \mathrm{~b}$ & $0,5 \pm 0,0 \mathrm{a}$ & $72,3 \pm 3,1 \mathrm{a}$ \\
$\mathbf{4}$ & $5,97 \pm 0,0 \mathrm{e}$ & $0,02 \pm 0,0 \mathrm{a}$ & $69,8 \pm 4,7 \mathrm{~b}$ & $0,5 \pm 0,1 \mathrm{a}$ & $63,6 \pm 1,4 \mathrm{a}$ \\
$\mathbf{5}$ & $6,00 \pm 0,0 \mathrm{c}$ & $0,02 \pm 0,0 \mathrm{a}$ & $70,9 \pm 0,1 \mathrm{a}$ & $0,6 \pm 0,3 \mathrm{a}$ & $65,8 \pm 1,0 \mathrm{a}$ \\
$\mathbf{6}$ & $6,03 \pm 0,0 \mathrm{a}$ & $0,02 \pm 0,0 \mathrm{a}$ & $70,9 \pm 0,8 \mathrm{a}$ & $0,5 \pm 0,0 \mathrm{a}$ & $70,3 \pm 0,6 \mathrm{a}$ \\
$\mathbf{7}$ & $6,02 \pm 0,0 \mathrm{~b}$ & $0,02 \pm 0,0 \mathrm{a}$ & $71,1 \pm 0,6 \mathrm{a}$ & $0,5 \pm 0,3 \mathrm{a}$ & $62,0 \pm 2,1 \mathrm{a}$ \\
$\mathbf{8}$ & $6,02 \pm 0,0 \mathrm{~b}$ & $0,02 \pm 0,0 \mathrm{a}$ & $68,2 \pm 0,3 \mathrm{~b}$ & $0,5 \pm 0,0 \mathrm{a}$ & $60,9 \pm 3,6 \mathrm{a}$ \\
$\mathbf{9}$ & $6,02 \pm 0,0 \mathrm{~b}$ & $0,02 \pm 0,0 \mathrm{a}$ & $69,4 \pm 0,2 \mathrm{~b}$ & $0,4 \pm 0,0 \mathrm{a}$ & $60,7 \pm 0,8 \mathrm{a}$ \\
\hline $\mathbf{1 0}$ & $5,98 \pm 0,0 \mathrm{~d}$ & $0,02 \pm 0,0 \mathrm{a}$ & $69,5 \pm 0,0 \mathrm{~b}$ & $0,5 \pm 0,1 \mathrm{a}$ & $51,4 \pm 2,2 \mathrm{a}$ \\
\hline
\end{tabular}

Médias seguidas da mesma letra, nas colunas, não diferem estatisticamente entre si, pelo Teste de Scott-Knott a $5 \%$ de probabilidade. F1, amido resistente; F2, polidextrose; F3, inulina; F4, 50\% amido resistente e $50 \%$ polidextrose; F5, 50\% amido resistente e $50 \%$ inulina; $\mathrm{F} 6,50 \%$ polidextrose e $50 \%$ inulina; $\mathrm{F} 7,68 \%$ amido resistente, $16 \%$ polidextrose e $16 \%$ inulina; $\mathrm{F} 8$, $16 \%$ amido resistente, $68 \%$ polidextrose e $16 \%$ inulina; $\mathrm{F} 9,16 \%$ amido resistente, $16 \%$ polidextrose e $68 \%$ inulina; $\mathrm{F} 10,33 \%$ amido resistente, $33 \%$ polidextrose e $33 \%$ inulina. Acidez Total (g ácido cítrico/100 g), Umidade (g/100 g), Cinzas (g/100 g), Fenólicos Totais (mg GAE/g de produto)

Houve diferença significativa para os valores de $\mathrm{pH}$ (Tabela 3 ) entre as formulações ( $\mathrm{p} \leq$ $0,05)$, que variaram de 5,95 a 6,03. A combinação de fibras alterou o $\mathrm{pH}$, porém mais estudos são necessários para verificar o motivo desta diferença. O estudo de Silva (2012) com adição de FOS ao sorvete obteve um pH médio de 7,10. Aykan et al. (2008) substituiu gordura por maltodextrina, inulina e oligofrutose parcial e totalmente em duas formulações distintas, obtendo pH de 6,44 e 6,42, respectivamente, sendo esses valores mais próximos aos desse estudo.

Os dados de sólidos solúveis, atividade antioxidante, sabor, consistência, doçura e impressão global foram submetidos a análise de metodologia de superfície de resposta por meio da regressão da superfície (Tabela 4) e uma equação para cada parâmetro foi desenvolvida. Foi ajustado um modelo quadrático para sólidos solúveis, atividade antioxidante, sabor e impressão global e um modelo linear para consistência e doçura.

Observa-se que de modo geral, a adição das fibras isoladas foram aquelas que mais influenciaram os parâmetros estudados, indicando que a adição destas fibras isoladamente provoca efeito linear positivo nestes parâmetros. Nota-se que, em relação aos atributos sensoriais avaliados (sabor, consistência, doçura e impressão global), a variável que mais afetou a resposta foi a inulina (valores maiores nas equações). Isso mostra que o uso de maiores concentrações de inulina faz com que os sorvetes sejam mais aceitos. 
TABELA 4 - MODELOS PREDITOS PARA DOS DADOS SENSORIAIS E FÍSICO-QUÍMICOS DOS SORVETES FUNCIONAIS ADICIONADOS DE KINKAN

\begin{tabular}{|c|c|c|}
\hline Atributos & Modelo Predito & $\mathbf{R}^{2}$ \\
\hline $\begin{array}{l}\text { Sólidos solúveis } \\
\text { ( }{ }^{\circ} \text { Brix) }\end{array}$ & $Y=23,47 X_{1}^{*}+30,43 X_{2}^{*}+30,36 X_{3}^{*}-0,79 X_{1} X_{2}+0,36 X_{1} X_{3}-1,06 X_{2} X_{3}$ & 0,96 \\
\hline 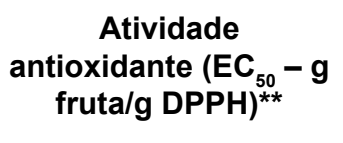 & $\begin{array}{c}Y=36251,9 X_{1}^{*}+12400,7 X_{2}+46378,7 X_{3}^{*}+169935,5 X_{1} X_{2}^{*}+ \\
65290,2 X_{1} X_{3}-43306,5 X_{2} X_{3}\end{array}$ & 0,91 \\
\hline Sabor & $Y=4,75 X_{1}^{*}+6,06 X_{2}^{*}+6,42 X_{3}^{*}-0,91 X_{1} X_{2}+0,22 X_{1} X_{3}+2,00 X_{2} X_{3}$ & 0,93 \\
\hline Consistência & $Y=6,12 X_{1}^{*}+6,9 X_{2}^{*}+7,07 X_{3}^{*}$ & 0,71 \\
\hline Doçura & $Y=5,30 X_{1}^{*}+6,41 X_{2}^{*}+6,76 X_{3}^{*}$ & 0,81 \\
\hline Impressão Global & $Y=5,50 X_{1}^{*}+6,28 X_{2}^{*}+6,64 X_{3}^{*}-0,58 X_{1} X_{2}+0,58 X_{1} X_{3}+1,67 X_{2} X_{3}$ & 0,95 \\
\hline
\end{tabular}

$\mathrm{X}_{1}$, amido resistente; $\mathrm{X}_{2}$, polidextrose; $\mathrm{X}_{3}$, inulina ${ }^{\star \star} \mathrm{DPPH}\left(\mathrm{EC} 50-\mathrm{g}\right.$ b.u../g DPPH) ${ }^{\star}$ significativo ao nível de 0,05

A curva de contorno da impressão global encontra-se na Figura 1.

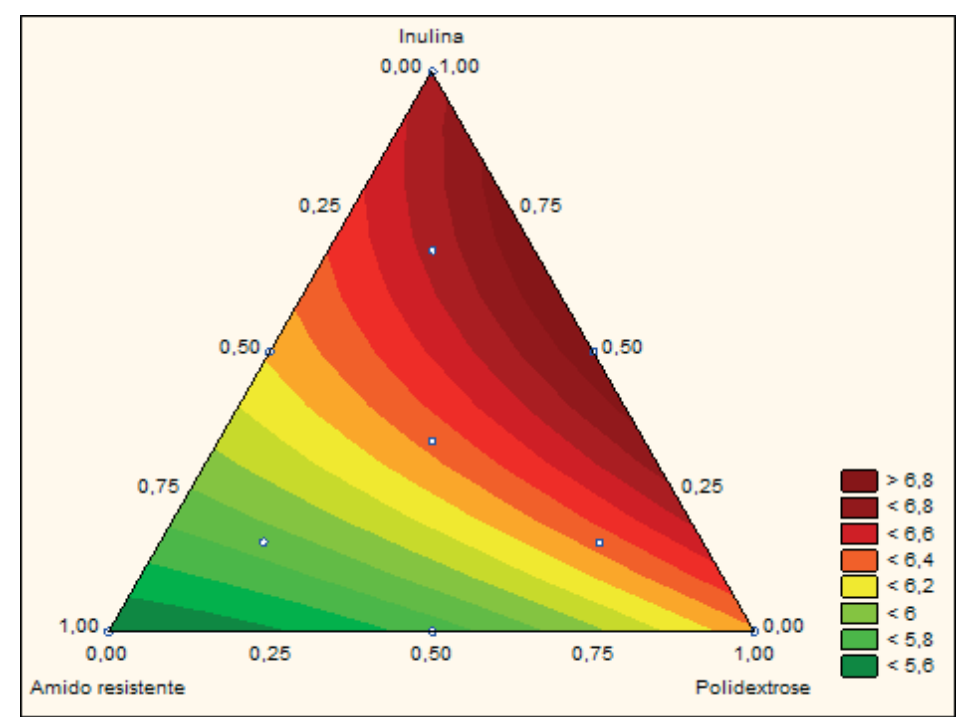

FIGURA 1. SUPERFÍCIE DE CONTORNO DO ATRIBUTO IMPRESSÃO GLOBAL DOS SORVETES FUNCIONAIS ADICIONADOS DE KINKAN

A avaliação sensorial analisada através da superfície de contorno mostrou que notas maiores que 6,8 foram adquiridas quando houve ausência de amido resistente e presença de polidextrose entre $0,25 \%$ a $0,75 \%$ e de inulina entre $0,25 \%$ a $0,75 \%$ (concentrações baseadas na substituição de $10 \%$ de gordura). 


\section{CONCLUSÕES}

O kinkan apresentou elevado teor de sólidos solúveis e baixa acidez, tanto na sua forma in natura, quanto processado, além de ser rico em antioxidantes. Essas características o fizeram ser uma boa escolha para o processamento de sorvete adicionado de fibras.

O sorvete funcional adicionado de kinkan apresentou boa aceitação sensorial, alta atividade antioxidante e elevado teor de fenólicos totais, sendo uma alternativa saudável e interessante para o mercado de alimentos funcionais. Em relação às fibras utilizadas, pode-se concluir que para maior aceitabilidade dos consumidores, deve-se utilizar apenas inulina $(0,25 \%$ a $0,75 \%)$ e polidextrose $(0,25 \%$ a $0,75 \%)$ nas formulações dos sorvetes.

\section{AGRADECIMENTOS}

Os autores agradecem à UFOP pelo apoio financeiro, à Gemacon® pela doação do mix de estabilizantes/emulsificantes e do amido resistente, à Nutramax® pela doação da polidextrose e à Metachem® pela doação da inulina.

\section{ABSTRACT}

\section{DEVELOPMENT OF FUNCTIONAL ICE CREAM ADDED EXOTIC FRUIT}

Currently, consumers have shown great concern in acquiring more nutritious and healthy products, with different flavors and, above all, safe for consumption. This is a market trend and, with the ice cream very much appreciated in Brazil, the aim of this study was to develop and optimize functional ice cream added kinkan. For this purpose, we used three dietary fibers (modified starch, polydextrose and inulin) isolated and in different combinations in order to obtain a product rich in fiber and bioactive compounds. It was found that the kinkan has good physico-chemical properties, both in its form as fresh and processed, in addition to having high antioxidant levels. These characteristics make the kinkan is a good choice to be added to ice cream rich in fiber. The functional ice cream with added kinkan demonstrated good acceptability, high antioxidant activity and high content of phenolic compounds, which makes it a healthy and interesting alternative to the functional food market. With regard to fibers used, one can conclude that, for better consumer acceptability, inulin should be added $(0.25 \%$ to $0.75 \%)$ and polydextrose $(0.25 \%$ to $0.75 \%)$ in formulation of ice cream.

KEY-WORDS: KINKAN, FUNCTIONAL ICE CREAM, DIETARY FIBER.

\section{REFERÊNCIAS}

1 ABIS - Associação Brasileira das Indústrias de Sorvete. Disponível em <http://www.abis.com.br/index.html>.

2 ACOSTA, O.; VÍQUEZ, F.; CUBERO, E. Optimization of low calorie mixed fruit jelly by response surface methodology. Food Quality and Preference.Barking, v.19, n.1, p.79-85, 2008.

3 AGÓCS, A.; NAGY, V.; SZABÓ, Z.; MÁRK, L.; OHMACHT, R.; DELI, J. Comparative study on the carotenoid composition of the peel and the pulp of different citrus species. Innovative Food Science \& Emerging Technologies, v.8, p.390-394, 2007.

4 A.O.A.C (Association of Official Analitical Chemistry). Official methods of analysis of the association of analytical chemistry, v.17, 2000.

5 AYKAN, V.; SEZGIN, E.; GUZEL-SEYDIM, Z. B. Use of fat replacers in the production of reduced-calorie vanilla ice cream. European Journal of Lipid Science and Technology, v.110, n.6, p.516-520, 2008. 
6 BARRECA, D.; BELLOCO, E.; CARISTI, C.; LEUZZI, U.; GATTUSO, G. Kumquat (Fortunella japonica Swingle) juice: Flavonoid distribuition and antioxidant properties. Food Research International, v.44, p.2190-2197, 2011.

7 BRASIL. MINISTÉRIO DA SAÚDE. AGÊNCIA NACIONAL DE VIGILÂNCIA SANITÁRIA. Regulamento técnico para fixação de identidade e qualidade de gelados comestíveis e, preparados para gelados comestíveis, pós para o preparo e bases para gelados comestíveis. Resolução RDC n. 266 de 22 de setembro de 2005. Diário Oficial da União, Poder Executivo, Brasília, DF, 2005.

8 CORNELL, J. A. Experiment with Mixtures: Design, Models and Analysis of Mixtures Data. John Wiley, New York, NY, 1983.

9 CQH - Centro de Qualidade em Horticultura - CEAGESP. (2000a). Classificação da Laranja (Citrussinensis, Osbeck). São Paulo: CEAGESP. (Folder). 2p

CQH - Centro de Qualidade em Horticultura - CEAGESP. (2000b). Classificação das Tangerinas. São Paulo: CEAGESP. (Folder). 2p

11 CQH - Centro de Qualidade em Horticultura - CEAGESP. (2000c). Classificação do limão (lima ácida) Tahiti (Citruslatifólia ,Tanaka).São Paulo: CEAGESP. (Folder). 2p

12 CUMMIGS, J. H; EDMOND, L. M.; MAGEE, E. A. Dietary carbohydrates and health: do we still need the fibre concept? Clinical Nutrition Supplements, v.1, n.2, p.5-17, 2004.

FERREIRA, D. F. Programa Sisvar: versão 3,04. Lavras: UFLA/DEX, 2000.

14 I.A.L. INSTITUTO ADOLFO LUTZ. Normas analíticas do Instituto Adolfo Lutz. Métodos químicos e físicos para análise de alimentos. 2d., São Paulo, v.1, 533p, 2005.

15 JAYAPRAKASHA, G. K.; MURTHY, C.; ETLINGER, M.; MANTUR, S. M.; PATIL, B. S. Radical scavenging capacities and inhibition of human prostate (LNCap) cell proliferation by Fortunella margarita. Food Chemistry, v.131, n.1, p.184191, 2012.

SILVA JÚNIOR, G. B.; ROCHA, L. F.; AMARAL, F. H. C.; ANDRADE, M. L.; NETO, R. F.; CAVALCANTE, I. H. L. Laranja-da-terra: fruta cítrica potencial para o Piaú. Semina: Ciências Agrárias, v.31, n.3, p.557-562, 2010.

17 LARRAURI, J. A.; RUPEREZ, P.; SAURA-CALIXTO, F. Effect of drying temperature on the stability of polyphenols and antioxidant activity of red grape pomace peels. Journal of Agricultural and Food Chemistry, v.45, p.1390-1393, 1997.

18 LISTA DE ESPÉCIES DA FLORA DO BRASIL. Jardim Botânico do Rio de Janeiro. Disponível em: < http://www. floradobrasil.jbrj.gov.br>.

19 MARSHALL, R. T.; ARBUCKLE, W. S. Ice cream. New York: International Thomson Publishing, 5a ed., 349p, 1996.

20 MOSCATTO, J. A.; PRUDÊNCIO-FERREIRA, S. H.; HAULY, M .C. O. Farinha de yacon e inulina como ingredientes na formulação de bolo de chocolate. Ciência e Tecnologia de Alimentos, v.24, n.4, p.634-640, 2004.

21 NASCIMENTO, V. E.; MARTINS, A. B. G.; HOJO R. H. Caracterização física e química de frutos de mamey. Revista Brasileira de Fruticultura, v.30, n.4, p.953-957, 2008.

22 PAUCAR-MENACHO, L. M.; SILVA, L. H.; BARRETO, P. A. A.; MAZAL, G.; FAKHOURI, F. M.; STEEL, C. J.; COLLARES-QUEIROZ, F. P. Desenvolvimento de massa alimentícia fresca funcional com a adição de isolado protéico de soja e polidextrose utilizando páprica como corante. Ciência e Tecnologia de Alimentos, v.28, n.4, p.767-778, 2008.

23 PENG, L.; SHEU, M.; LIN, L.; WU, C.; CHIANG, H.; LIN, W.; LEE, M.; CHEN, H. Effect of heat treatments on the essential oils of kumquat (Fortunella margarita Swingle). Food Chemistry, v.136, p.532-537, 2013.

24 PEREIRA, A. C. S. Qualidade, compostos bioativos e atividade antioxidante total de frutas tropicais e cítricas produzidas no Ceará. 120p, 2009. Dissertação (Mestrado em Ciência e Tecnologia de Alimentos) - Embrapa Agroindústria Tropical, Fortaleza.

25 RESENDE, J. T. V.; MORALES, R. G. F.; FARIA, M. V.; RISSINI, A. L. L.; CAMARGO, L. K. P.; CAMARGO, C. K. Produtividade e teor de sólidos solúveis de frutos de cultivares de morangueiro em ambiente protegido. Horticultura Brasileira, v.28, p.185-189, 2010.

26 ROGEZ, H.; BUXANT, R.; MIGNOLET, E.; SOUZA, J. N. S.; SILVA, E. M.; LARONDELLE, Y. Chemical composition of the pulp of three typical Amazonian fruits: araçá-boi (Eugenia stipitata), bacuri (Platonia insignis) and cupuaçu (Theobroma grandiflorum). European Food Research and Technology, v.218, p.380-384, 2004. 
27 RUFINO, M. S. M.; ALVES, R. E.; BRITO, E. S.; MORAIS, S. M.; SAMPAIO, C. G.; PÉREZ-JIMÉNEZ, J.; SAURACALIXTO, F. D. Metodologia científica: determinação da atividade antioxidante total em frutas pela captura do radical livre DPPH. Comunicado Técnico. Empresa Brasileira de Pesquisa Agropecuária, 2007.

28 SALES, R. L.; VOLP, A. C. P.; BARBOSA, K. B. F.; DANTAS, M. I. S.; DUARTE, H. S.; MINIM, V. P. R. Mapa de preferência de sorvetes ricos em fibras. Ciência e Tecnologia de Alimentos, v.28, p.27-31, 2008.

29 SADEK, E. S.; MAKRIS, D. P.; KEFALAS, P. Polyphenolic composition and antioxidant characteristics of Kumquat (Fortunella margarita) peel fractions. Plant Food Human Nutrition, v.64, p.297-302, 2009.

30 SEOLIN, V. J.; SCAPIM, M. R. S.; PIERETTI, G. G.; TONON, L. A. C.; MADRONA, G. S. Substituição de sacarose por frutooligosacarídeo em sorvete. Revista Brasileira de Tecnologia Agroindustrial, v.7, n.2, p.1062-1073, 2013.

31 STONE, H.; SIDEL, J. L. Sensory Evaluation Practices. London: Academic Press, 1985. 338p.

32 SHUN-ZHEN, L.; HONG-XING, L.; LI-XIA, Z.; YAN-MIN, H. Comparative analysis on chemical components in essential oils from the leaves and fruit peels of Fortunella margarita. Medicinal Plant, v.3, n.1, p.43-46, 2012.

33 SILVA, V. M. Sorvete light com fibra alimentar: desenvolvimento, caracterização físico-química, reológica e sensorial. 147p. 2012. Dissertação (Mestrado em Ciência e Tecnologia de Alimentos) - Universidade Federal de Viçosa, Viçosa.

34 TACO. Tabela brasileira de composição de alimentos/NEPA. UNICAMP.ed. 4.161 p. Campinas, 2011.

35 TAN, S.; LI, M.; DING, X.; FAN, S.;GUO, L.; GU, M.;ZHANG, Y.; FENG, L.; JIANG, D.; LI, Y.; XI, W.; HUANG, C.;ZHOU, Z. Effects of Fortunella margarita Fruit Extract on Metabolic Disordes in High-Fat Diet-Induced Obese C57BL/6 Mice. PlosOne v.9, n.4, 2014.

36 UCHOA, A. M. A.; COSTA, J. M. C.; MAIA, G. A.; SILVA, E. M. C.; CARVALHO, A. F. F. U.; MEIRA, T. R. Parâmetros físico-químicos, teor de fibra bruta e alimentar de pós alimentícios obtidos de resíduos de frutas tropicais. Segurança Alimentar e Nutricional, v.15, n.2, p.58-65, 2008.

37 VASCO, C.; RUALES, J.; KAMAL-ELDIN, A. Total phenolic compounds and antioxidant capacities of major fruits from Ecuador. Food Chemistry, v.111, n.4, p.816-823, 2008.

38 VILAS BOAS, B. M.; NUNES, E. E.; FIORINI, F. V. A.; LIMA, L. C. O.; VILAS BOAS, E. V. B.; COELHO, A. H. R. Avaliação da qualidade de mangas "Tommy Atkins" minimamente processadas. Revista Brasileira de Fruticultura, v.26, n.3, p.540-543, 2004

39 WALTER, M.; SILVA, L. P.; EMANUELLI, T. Amido resistente: características físico-químicas, propriedades fisiológicas e metodologias de quantificação. Ciência Rural, v.35, n.4, p.974-980, 2005.

40 WATERhouse, A. L. Polyphenolics: Determination of total phenolics. In: WROLSTAD, R. E. Current Protocols in Food Analytical Chemistry, v.11, p.111-111, 2002. 